\title{
Philosophical Proofs Against Common Sense
}

\author{
Bryan Frances
}

Forthcoming in Analysis

\begin{abstract}
Many philosophers are sceptical about the power of philosophy to refute commonsensical claims. They look at the famous attempts and judge them inconclusive. I prove that even if those famous attempts are failures, there are alternative successful philosophical proofs against commonsensical claims. After presenting the proofs I briefly comment on their significance.
\end{abstract}

\section{The Initial Proof}

Consider the following $\mathrm{C}$ sentences.

- Anyone with less than $1 c$ is not rich.

- If anyone with less than $1 c$ is not rich, then anyone with less than $2 c$ is not rich.

- If anyone with less than $\left(10^{14}-1\right) c$ is not rich, then anyone with less than $10^{14} \zeta$ is not rich.

- It's not the case that anyone with less than $10^{14} \mathrm{C}$ is not rich.

Surely there's a way of interpreting all but the final sorites $C$ sentences so that one derives from them, using just Modus Ponens (MP), 'Anyone with less than $10^{14} \mathrm{C}$ is not rich'. The final $\mathrm{C}$ is the negation of that very claim. To have MP apply to the Cs in the obvious way to make the derivation, we do two things.

First, interpret the Cs so that they involve the logical forms they certainly seem to involve. For instance, all but the first and last Cs are material conditionals, by design. Second, insure that 'anyone', 'is with', and 'is rich' are univocal throughout the Cs so MP applies in the usual way. In order to preserve univocality replace or analyze 'anyone' with something like 'any adult person in Queens, New York in 1999 who is single with no dependents'. Similarly, we can precisify 'is with $\mathrm{NC}^{\prime}$ ' with something involving a particular measure of wealth. Or, we can quantify over such measures.

Sorites arguments need not turn on terms with obvious context dependency such as 'is rich'. They can turn on predicates such as 'is a pumpkin'. For instance, we can do a sorites series on 'There is a pumpkin by the tree' by proceeding through a series of cases that diminish the pumpkin atom by atom starting from its top (so the sentence starts out true and ends up false). Or, we can have the series focus on the term 'by': the neighboring cases in the series differ by having the pumpkin a yoctometer farther from the tree, so 'There is a pumpkin by the tree' goes from true (the pumpkin is on the ground touching the tree) to false (the pumpkin is as far as you like). We can even use proper names such as 'Bertrand Russell'. There are other standard ways of getting around context-dependency. 
The term 'commonsensical' admits of multiple readings, one of which relativizes to group and time, so 'The Earth is round' is commonsensical for some groups and times but not others. 'MP is truth-preserving' is commonsensical for those who know logic but not to most other groups (just truth-preserving; in this paper I make no use of stronger notions such as 'truth-preserving in virtue of logical form'). Stipulate that $\mathrm{P}$ is commonsensical at a time for a certain large community iff virtually everyone in that community at that time who understands $\mathrm{P}$ well is strongly disposed to give $\mathrm{P}$ a high credence. In this paper I'm concerned with common sense amongst contemporary philosophers. Some clarifications are in order.

First, the definition does not count $\mathrm{N}$, 'There are twice as many positive integers as there are even positive integers', as commonsensical amongst philosophers. Although $\mathrm{N}$ is intuitive if you put your mind in the right "frame", it doesn't count as commonsensical for contemporary philosophers, in my sense, because even though we can still feel the intuitive pull of $\mathrm{N}$, the vast majority of us will reject it since we have heard at least a bit about elementary transfinite number theory.

Second, one doesn't have to be a successful researcher on P in order to "understand P well", in the sense I'm using that phrase. For instance, I'm neither a logician nor a philosopher of logic but I understand 'MP is truth-preserving' well. I've taught logic several times, I once took a class in the philosophy of logic, and I've studied a few articles in the field.

Here is the first premise of my first proof:

1. There exists an interpretation and group of familiar elementary inference rules of sentential logic such that (i) each so-interpreted $C$ is commonsensical, and (ii) from just the so-interpreted Cs there is a derivation of a pair of contradictory claims using just those rules of inference.

Virtually no one will deny that there is an interpretation W of the sorites Cs so that clause (ii) holds: one can derive a pair of contradictory claims from them using MP. It's the interpretation that went through your mind when you first read the Cs and immediately saw how to apply MP to them in the perfectly ordinary way. Hence, if (1) isn't true, then under that $W$ interpretation-one that allows for the derivation-at least one $\mathrm{C}$ is not commonsensical (amongst philosophers); that would be clause (i) failing to be satisfied. But what on earth is that specific $C$ saying if it's not commonsensical?

The first and last Cs are obviously commonsensical no matter how one interprets them. Thus, if one of the Cs isn't commonsensical under W, it has to be one of the "middle" Cs that fails to be commonsensical. But we know perfectly well that under $W$ each of the middle $C s$ has the following form:

If anyone with less than $N C$ is not rich, then anyone with less than $(N+1) c$ is not rich.

Anyone competent in elementary logic can see that since each middle $\mathrm{C}$ is a material conditional with universal generalizations as antecedents and consequents (by design), in order for it to be false, everyone with up to $\mathrm{N} C \mathrm{C}$ has to be not rich but someone with from $\mathrm{N} C$ to just under $(\mathrm{N}+1) \boldsymbol{C}$ is rich. And anyone who knows about the large numbers of people in the world and their extreme spread in wealth knows that if a middle $\mathrm{C}$ is false, then there are two people who differ by only a few cents and yet just one of them is rich. Or take a term such as 'pumpkin': if a middle C is false, then there are two pumpkinish things, that differ in just a few atoms on their respective tops, but only one of them is a pumpkin. A third example: 'The restaurant is a short walk from here' goes from true to false in the space of a yoctometer. Those are 
clearly against common sense for philosophers: the vast majority of us will believe their negations when asked to evaluate them. Again, one of the middle Cs might be false but that doesn't prevent it from being commonsensical: it's a sociological fact that the sorites Cs are commonsensical amongst philosophers who understand them well.

If all the sorites $C s$ are true under an interpretation $W$ and there is a derivation from them of a pair of contradictory claims using MP alone, but MP isn't truth-preserving when applied to the $W$-interpreted Cs, then ' $\mathrm{MP}$ is truth-preserving when applied to the $\mathrm{W}$-interpreted $\mathrm{Cs}^{\prime}$ ' is both untrue and yet commonsensical. The first conjunct of the consequent is a trivial logical consequence of the antecedent plus an obviously true instance of the schema 'If $\sim P$, then ' $P$ ' isn't true'. The other conjunct of the consequent is entailed by the utterly bland claim that 'MP is truth-preserving when applied to the Winterpreted Cs' is commonsensical (amongst philosophers). Premise (2) is a generalization of this idea.

2. For any interpretation $\mathrm{W}$ and group of familiar elementary inference rules $\mathrm{R}$ of sentential logic, if (i) and (ii) from (1) are true of W/R/the Cs but at least one of the Rs is not truth-preserving applied to the $\mathrm{W}$-interpreted Cs, then 'Each of the Rs is truth-preserving when applied to the Winterpreted Cs' isn't true but commonsensical.

Premise (3) is like (2) in being entailed by a bland thought: negations of contradictions are commonsensical.

3. For any interpretation $W$ and group of familiar elementary inference rules $R$ of sentential logic, if (i) and (ii) from (1) are true of $W / R /$ the $C s$, each $R$ is truth-preserving when applied to the $W$ interpreted Cs, and all the W-interpreted Cs are true, then (since the derivation of a pair of contradictory claims has all true premises and uses nothing but rules that are truth-preserving when applied to the $\mathrm{W}$-interpreted Cs) the negation of a contradiction isn't true but commonsensical.

(4) is a logical truth but helpful to make explicit.

4. For any interpretation $W$ and group of familiar elementary inference rules $R$ of sentential logic, if (i) and (ii) from (1) are true of $W / R /$ the $C s$ (which entails that each $W$-interpreted $C$ is commonsensical), each $\mathrm{R}$ is truth-preserving when applied to the $\mathrm{W}$-interpreted $\mathrm{Cs}$, and at least one of the W-interpreted Cs isn't true, then at least one of the W-interpreted Cs isn't true but commonsensical.

(5) is an obvious logical consequence of (1)-(4), and (6) is an obvious logical consequence of (5).

5. Hence, by (1)-(4) there exists an interpretation $W$ and group of familiar elementary inference rules $\mathrm{R}$ of sentential logic such that either (a) 'Each $\mathrm{R}$ is truth-preserving when applied to the $\mathrm{W}$ interpreted Cs' isn't true but commonsensical (this is from (2)), (b) a negated contradiction isn't true but commonsensical (this is from (3)), or (c) at least one of the W-interpreted Cs isn't true but commonsensical (this is from (4)).

6. Hence, by (5) some claim (from either (a), (b), or (c) in (5)) isn't true but commonsensical.

(1)-(6) is a non-formal philosophical proof of the proposition a commonsensical claim isn't true. 
The only way for a philosopher to be aware of (1)-(6) and (competently) reject its conclusion is to reject at least one of (1)-(4). But (4) is an obvious logical truth and (2) and (3) are obviously entailed by the obviously true claims 'Negations of contradictions are commonsensical' and 'It's commonsensical that the derivation's familiar elementary rules of inference (for the sorites, it's just MP) are truth-preserving when applied to the $W$-interpreted Cs' (plus an application of 'If $\sim P$, then ' $P$ ' isn't true'). Hence, the only (competent) way to reject the soundness of (1)-(6) is to reject the truth of (1). But we saw that (1) is about as certain as any philosophical claim ever gets.

Hence, non-formal philosophy refutes a portion of common sense (for approximately contrary attitudes see Lycan 2001 and 2019, Kelly 2008, Schaffer 2009, Lewis 1973, Fine 2001, and Gupta 2006; for supportive claims see Frances 2019 and Schwitzgebel 2014 and 2017).

\section{The Revenge Proof}

Suppose that contrary to my argument of $\$ 1$, there is an excellent philosophical argument that (1) isn't true. So, we are now assuming that there is an argument of the form ' $P_{1}-P_{n}$; thus, (1) isn't true', where the Ps are the premises of this amazing argument that (1) isn't true. Incredibly, the existence of this ' $P_{1}-P_{n}$; thus, (1) isn't true' argument will not help avoid my thesis that non-formal philosophy refutes a portion of common sense.

Note first that virtually all philosophers who understand the sorites paradox believe the following sociological proposition about their own community: virtually all its members think that there's a way of understanding the Cs so that they are commonsensical and collectively inconsistent. Being in the community of philosophers who know the sorites paradox well (philosophers of language) almost always means knowing a good deal about what others in the community think about the paradox; we are social creatures, after all. So, philosophers familiar with the paradox overwhelmingly think that virtually everyone familiar with the paradox thinks there's an interpretation of the Cs so that they are commonsensical and jointly inconsistent.

This shows that (1) is commonsensical relative to the group of people today who understand it well. Now do an exercise.

- Let $\mathrm{P}$ be '( 1 ) is commonsensical'.

- Take the hypothesized argument that (1) isn't true-the argument ' $P_{1}-P_{n}$; thus, (1) isn't true'and modify it so it becomes ' $P, P_{1}-P_{n}$; thus, $((1) \text { isn't true } \& P)^{\prime}$. That is, add the just-defined $P$ to its premises and make the new conclusion a conjunction of the old conclusion '(1) isn't true' and $P$. Clearly, that argument is no worse than the original argument ' $P_{1}-P_{n}$; thus, (1) isn't true', as we are in effect merely adding $P$ to both the premises and the conclusion.

- Note that the new conclusion '(1) isn't true \& P' obviously entails, by existential generalization, 'Some claim isn't true but commonsensical'.

- Construct the second new argument ' $\mathrm{P}, \mathrm{P}_{1}-\mathrm{P}_{\mathrm{n}}$; thus, some claim isn't true but commonsensical'. Obviously, that argument is no worse than the one we started with, ' $P_{1}-P_{n}$; thus, (1) isn't true'.

Here is the revenge proof that non-formal philosophy refutes a portion of common sense: 
I. If (1) is true, then (since (1)-(6) is clearly valid and philosophical, and all its other premises are clearly true) there is an excellent philosophical argument that some claim isn't true but commonsensical.

II. If (1) isn't true, then there is an excellent philosophical argument ' $P_{1}-P_{n}$; thus, (1) isn't true'. (That is, if (1) isn't true, then is an excellent philosophical argument for that fact. This premise is not at all trivial, but it will be revealed below to be dialectically apt.)

III. Let $\mathrm{P}$ be '(1) is commonsensical'. $\mathrm{P}$ is true, as shown above by reflecting on what philosophers think about each other.

IV. If $P$ is true and there is an excellent philosophical argument ' $P_{1}-P_{n}$; thus, (1) isn't true', then there is an excellent philosophical argument ' $P, P_{1}-P_{n}$; thus, some claim isn't true but commonsensical'. (I showed this in the above exercise.)

V. If there is an excellent philosophical argument ' $P, P_{1}-P_{n}$; thus, some claim isn't true but commonsensical', then there is an excellent philosophical argument that some claim isn't true but commonsensical.

VI. Hence, by (II)-(V), if (1) isn't true, then there is an excellent philosophical argument that some claim isn't true but commonsensical.

VII. Hence, by (I) \& (VI), there is an excellent philosophical argument that some commonsensical claim isn't true.

The philosopher who thinks philosophy doesn't refute a portion of philosophical common sense has to reject the soundness of (1)-(6). That means claiming (1) isn't true, since the rest of the argument is indisputable. Since she holds that (1) isn't true, she probably thinks there is an excellent philosophical argument against (1) - in fact, it's highly likely that she thinks she could tell us in detail about that excellent argument against (1). After all, (1) is highly intuitive, so if she thinks it's not true, she will very probably think she has an excellent argument against it. Hence, she will agree with premise (II). Since by hypothesis she denies (VII), she has to deny (III), since the rest of the obviously valid (I)-(VII) argument is indisputable. But to deny (III) is to deny a stubborn, empirical, non-normative fact about the views of contemporary philosophers: the fact that the vast majority of philosophers familiar with the sorites think that the Cs can have their obvious logical characteristics and still be commonsensical.

\section{Philosophical Significance}

The proofs need not use the sorites. Many philosophical problems can be used to generate a set of $C$ claims that have very similar consequences. Making lists of Cs for new proofs is a matter of patience. All we need is a set of commonsensical claims that under a commonsensically available interpretation uses commonsensically truth-preserving inference rules to derive the negation of a commonsensical claim. Some proofs will be significantly stronger than others. As one would expect, the liar paradox is similar to the sorites in that it's perfectly suited to generate extremely strong proofs. In addition, one can use the Problem of the Many:

- There is at least one tree in my backyard. 
- Every tree in my backyard is composed of chemical atoms.

- For all $\mathrm{x}$, if $\mathrm{x}$ is a tree in my backyard that is composed of chemical atoms, then there is at least one plurality of chemical atoms that composes it. (Some would say, with reason, that there is exactly one such plurality.)

- For all $x$ and $y$, if $x$ is a plurality of chemical atoms, $y$ is a tree in my backyard, and $x$ composes $y$, then there exists $w$ and $z$ such that $w$ is a plurality of chemical atoms, $x$ and $w$ differ in members, $z$ is a tree in my backyard, and $w$ composes $z$. (The two large pluralities (e.g. about $10^{30}$ chemical atoms in each) are literally about $99.9999999999999999999999999999 \%$ overlapping and are, to all appearances, equally good candidates for composing a tree.)

- For all $x$ and $y$, if $x$ and $y$ are pluralities of chemical atoms, $x$ and $y$ differ in members, and there are trees in my backyard $\mathrm{t} 1$ and $\mathrm{t} 2$ such that $\mathrm{x}$ composes $\mathrm{t} 1$ and $\mathrm{y}$ composes $\mathrm{t} 2$, then $\mathrm{t} 1 \neq \mathrm{t} 2$.

- There is at most one tree in my backyard.

Although these Cs are commonsensical, some of them are not as commonsensical as those for the sorites or liar.

Although we might not know which commonsensical claims are refuted, we know they are in four categories (with overlap and borderline cases):

Everyday Life Claims: 'Some people are rich', 'There are trees in North America'.

Elementary Logic Claims: 'Modus ponens is truth-preserving', 'Contradictions aren't true'.

Philosophical Claims: 'If a tree is composed of atoms, then there is a group of atoms that composes it', 'It's not the case that: anyone with less than $€ 65,000$ is not rich but someone with less than $€ 65,001$ is rich' (or some other amount of money), 'If 'A isn't true' is true, then $A$ isn't true' (from the liar).

Interpretation Claims: 'Some interpretation of the sorites Cs has them commonsensical and functioning as the only premises of a derivation of a contradiction using just familiar elementary inference rules'.

If there is a proof $P$ that there exists a false claim $K$ that the vast majority of people today who understand $\mathrm{K}$ well are strongly disposed to assign a high credence to, then $\mathrm{P}$ is philosophically significant for those people. Hence, we have shown that there are philosophically significant informal philosophical proofs. To that extent, philosophy achieves real results.

Some philosophers will think the Everyday Life category is the most significant one (perhaps that is the best way to interpret the defences of common sense in Moore 1925, Lycan 2001 and 2019, Kelly 2008, Schaffer 2009, Lewis 1973, Fine 2001, and Gupta 2006). However, whether it's significant that a commonsensical claim $\mathrm{K}$ isn't true might depend on whether $\mathrm{K}$ is paired with a closely connected truth that does the work that $\mathrm{K}$ was supposed to do. For instance, students in metaphysics classes often insist that compositional nihilism (the view that there are no proper parts) is merely superficially counterintuitive. After all, according to (the simple version of) nihilism although there are no trees in North America, since if they existed they would have proper parts, there are a great many tightly-knit groups of simples arranged tree-wise, and those individual groups are located precisely where and only 
where common sense says the individual trees are located. Refuting 'There are trees in North America' might not be as significant as commonly thought despite the fact that it falls into the Everyday Life category. It depends on (i) whether there are truths paired with the falsehoods, and (ii) whether the fact of such pairings diminishes the significance of the refutation.

Since there are many philosophical problems that can generate excellent arguments of the forms of (1)(6) and (I)-(VII), there are many false commonsensical claims. Suppose almost all of them are in the latter three categories (and, perhaps, there is virtually nothing in the first category). The philosophers who think philosophy doesn't refute common sense can be charitably interpreted as claiming that philosophy doesn't refute claims in the Everyday Life category. On the one hand, if they are right, then claims from everyday life have been spared. But that means we have missed (i) the lack of soundness of the simplest elementary inference rules, (ii) the falsehood of many standard philosophical claims such as 'If a tree is composed of atoms, then there is a group of atoms that composes it', and/or (iii) the falsehood of empirical platitudes such as 'Some interpretation of the sorites Cs has them commonsensical and functioning as the sole premises of a derivation of a contradiction using just familiar elementary inference rules'. Hence, that means most of us are bad at philosophy, since a great many of us endorse those false claims in our work. Flatter yourself as one of the exceptions; then focus on the unwashed masses for the purposes of this paper. In another paper I examine the epistemic consequences.

On the other hand, if many of the false claims are in the first category, then two interesting results follow. First, everyday common sense contains many remarkable falsehoods refuted by philosophy. Second, the Moorean move made so often in philosophy-when you encounter what you realize to be a philosophical argument that goes radically against everyday common sense, retain your commonsensical belief and conclude that the philosophical argument is unsound-is unreliable. In fact, it's unreliable even if the false claims are in the other categories, since the move is made in philosophical discourse constantly. ${ }^{1}$

\section{References}

Frances, B. 2019. The philosopher's doom: unreliable at truth or unreliable at logic. In The Mystery of Skepticism, eds. Ted Poston and Kevin McCain, 187-99. Leiden: Brill.

Gupta, A. 2006. Empiricism and Experience. Oxford: OUP Press.

Fine, K. 2001. The question of realism. The Philosophers' Imprint 1: 1-30.

Kelly, T. 2008. Common sense as evidence: against revisionary ontology and skepticism. In Midwest Studies in Philosophy: Truth and Its Deformities, eds. French and Wettstein, 53-78. Blackwell.

Lewis, D. 1973. Counterfactuals. Cambridge, MA: Harvard University Press.

Lycan, W. 2001. Moore against the New Skeptics. Philosophical Studies 103: 35-53.

Lycan, W. 2019. On Evidence in Philosophy. Oxford: OUP.

\footnotetext{
${ }^{1}$ Thanks to John Greco, Eric Schwitzgebel, Jonathan Matheson, David Christensen, and an Analysis referee for helpful comments.
} 
Moore, G. E. 1925. A defence of common sense. In Contemporary British Philosophy (2nd series), ed. J. Muirhead, 193-223. London: George Allen \& Unwin.

Schaffer, J. 2009. On what grounds what. In Metametaphysics: New Essays on the Foundations of Ontology, eds. Chalmers, Manley, and Wasserman. Oxford: OUP Press.

Schwitzgebel, E. 2014. The crazyist metaphysics of mind. Australasian Journal of Philosophy 92: 66582.

Schwitzgebel, E. 2017. 1\% skepticism. Noûs 51: 271-90. 